Pros

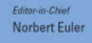

Journal of Nonlinear Mathematical Physics

ISSN (Online): 1776-0852 ISSN (Print): 1402-9251

Journal Home Page: https://www.atlantis-press.com/journals/jnmp

\title{
Invariant functions and contractions of certain types of Lie algebras of lower dimensions
}

J.M. Escobar, J. Núñez, P. Pérez-Fernández

To cite this article: J.M. Escobar, J. Núñez, P. Pérez-Fernández (2018) Invariant functions and contractions of certain types of Lie algebras of lower dimensions, Journal of Nonlinear Mathematical Physics 25:3, 358-374, DOI:

https://doi.org/10.1080/14029251.2018.1494705

To link to this article: https://doi.org/10.1080/14029251.2018.1494705

Published online: 04 January 2021 


\title{
Invariant functions and contractions of certain types of Lie algebras of lower dimensions
}

\author{
J.M. Escobar \\ Dpto. de Geometría y Topología. Facultad de Matemáticas. Universidad de Sevilla \\ Calle Tarfia, s/n, 41012 Sevilla \\ pinchamate@gmail.com \\ J. Núñez \\ Dpto. de Geometría y Topología. Facultad de Matemáticas. Universidad de Sevilla \\ Calle Tarfia, s/n, 41012 Sevilla \\ jnvaldes@us.es \\ P. Pérez-Fernández \\ Dpto. de Física Aplicada III, Escuela Técnica Superior de Ingeniería. Universidad de Sevilla \\ Camino de los Descubrimientos, s/n, 41092 Sevilla \\ pedropf@us.es
}

Received 24 July 2017

Accepted 4 February 2018

\begin{abstract}
In this paper, we deal with contractions of Lie algebras. We use two invariant functions of Lie algebras as a tool, named $\psi$ and $\varphi$ function, respectively, which have a great application in Physics due to their remarkable properties. We focus the study of these functions in the frame of the filiform Lie algebras, trying to extend to these algebras some of the properties of such functions over semi-simple Lie algebras.
\end{abstract}

Keywords: Contractions; invariant functions $\psi$; filiform Lie algebras.

2000 Mathematics Subject Classification: 17B30, 17B40, 17 B51.

\section{Introduction}

Many modern physical theories have a powerful mathematical framework underlying, in which different types of Lie algebras play a very important role. Specifically, Lie algebras (Sophus Lie Norwegian mathematician, 1842-1899) constitute certainly one of the most useful in order to describe symmetries underlying in these physical theories. As a example, it can be highlighted the importance that the Virasoro and Kac-Moody algebras have, among others, in the development of the Superstring Theory. Also, Lie algebras are essential in Conformal Field Theory, in which semi-simple Lie algebras guarantee the existence of a non-degenerate Killing form, which in turn allows to build vertex algebras, which are important in this theory [7]. But it is possible that the better known example is the application of Lie algebras to the celebrated Standard Model of particle physics, which describes the internal symmetries of the unitary product group $S U(3) \times S U(2) \times U(1)$.

The study of other physical concepts has significantly been enhanced by the limit processes which allow us to relate Lie algebras among themselves. These processes were first investigated by Segal [21] in 1951, although, as it has been just noted, they are currently being widely used. Two are the better known examples of these processes. The first of them involves the connection between 
classical mechanics and relativistic mechanics, with their respective Poincaré symmetry group and Galilean symmetry group. The second one is the limit process by which quantum mechanics is contracted to classical mechanics, when $\hbar \rightarrow 0$, which actually corresponds to a contraction of the Heisenberg algebra to the abelian algebra of the same dimension. Remember also that classical mechanics is a limiting case of relativistic mechanics. Hence the group of the former, the Galilean group, must be in some sense a limiting case of the relativistic mechanics' group. This means that the representations of the Galilean symmetry group must be limiting cases of the representations of the Poincaré symmetry group (see [15] for further information).

There also exist many works on contractions of algebras that address both the physical aspect of the applications of these contractions and the mathematical aspect focused in the study of the algebraic properties of these contractions. Indeed, after Segal, the concept of limit process between physical theories in terms of contractions of their associated symmetry groups was formulated by Erdal Inönü and Eugene Wigner [9, 10], who introduced the so-called Inönü-Wingner contractions or IW- contractions. Later, Saletan [20] studied a more general class of one-parameter contractions, for which the elements of the corresponding matrices are one-degree polynomials with respect to the contraction parameter (in fact, WI-contractions are a subclass of Saletan contractions). Other extensions of the IW- contractions are, for instance, the generalized Inönü-Wigner contractions, introduced by Melsheiner [4], the parametric degenerations [2,3], very used in the Algebraic Invariants Theory, and the singular contractions [10]. Other relevant papers that can be cited on this topic are $[6,11,13,14,16,23,24]$ and [25].

Due to these reasons, the main goals of this paper are two: the first, to study the proper contractions of filiform Lie algebras of lower dimensions. At this respect we obtain, according to notation by Hrivnák and Novotný [18], that there is a proper contraction from the filiform Lie algebra $\mathfrak{f}_{3}$ both to the algebra $3 \mathfrak{g}_{1}$ and the algebra $\mathfrak{g}_{3,2}$, whereas there exists no proper contraction either between $\mathfrak{f}_{3}$ and $\mathfrak{g}_{3,1}$, or $\mathfrak{f}_{3}$ and $\mathfrak{g}_{3,3}$. We also prove that there is no proper contraction from a filiform Lie algebra to a Heisenberg algebra.

The second goal is to compute the invariant functions $\psi$ and $\varphi$, introduced in 2007 by Hrivnák and Novotný [17], for the case of these filiform Lie algebras of lower dimensions. The reason for dealing with algebras of lower dimensions is because there are no complete classifications of Lie algebras of dimensions greater than 5 and, besides, few invariant for them are known. Therefore, the use of these invariant functions can be considered as a tool to get advances in the knowledge of these algebras, particularly in the study of contractions.

To finish this introduction, we would like to explain the motivations for dealing with filiform Lie algebras in particular. These algebras were introduced by M. Vergne in the late 60's of the past century [22], although before that, Blackburn had already studied the analogous class of finite p-groups and used the term maximal class to call them, which is also now used for Lie algebras. Vergne showed that, within the variety of nilpotent Lie multiplications on a fixed vector space, nonfiliforms can be consigned to small-dimensional components. Besides, filiform Lie algebras are the most structured algebras within the nilpotent Lie algebras, which allow us to use and study them easier than other Lie algebras (an historical evolution of Lie algebras in general can be checked in [1]).

Furthermore, in spite of filiform Lie algebras belong to a subclass of nilpotent Lie algebras, in the current literature there is not any difference among the values obtained of the invariant functions for filiform nilpotent Lie algebras and non-filiform nilpotent Lie algebras. For this reason, one of the main purposes of this paper has been to highlight the filiform cases among the rest of nilpotent 
Lie algebras in order to establish that difference and thereby facilitate the work of future researches in this topic. Particularly, the term filiform is not mentioned by the authors of the reference [16] through all the paper.

The structure of the paper is as follows: in Section 1 we give some preliminaries on Lie algebras in general and on filiform Lie algebras, in particular. Sections 2, 3 and 4 are devoted to extend some results by Hrivnák and Novotný $[17,18]$ to the case of filiform Lie algebras of dimensions 3, 4 and 5, respectively. Proper contractions between Heisenberg algebras and filiform Lie algebras are studied in Section 5 and finally, we point out certain conclusions about the results that we have found in the last section.

\section{Preliminaries}

We show in this section some preliminaries on filiform Lie algebras, invariant functions in Lie algebras and proper contractions of Lie algebras.

\subsection{Preliminaries on filiform Lie algebras}

In this subsection we show some preliminaries on Lie algebras in general and on filiform Lie algebras in particular. For a further review on these topics, the reader can consult [8].

An $n$-dimensional Lie algebra $\mathfrak{g}$ over a field $K$ is an $n$-dimensional vector space over $K$ endowed with a second inner law, named bracket product, which is bilinear and anti-commutative and which satisfies the Jacobi identity

$$
J(u, v, w)=[u,[v, w]]+[v,[w, u]]+[w,[u, v]]=0, \text { for all } u, v, w \in \mathfrak{g} .
$$

The center of $\mathfrak{g}$ is the set $Z(\mathfrak{g})=\{u \in \mathfrak{g} \mid[u, v]=0$, for all $v \in \mathfrak{g}\}$. The Lie algebra is said to be abelian if $[u, v]=0$, for all $u, v \in \mathfrak{g}$.

Two Lie algebras $\mathfrak{g}$ and $\mathfrak{h}$ are isomorphic if there exists a vector space isomorphism $f$ between them such that

$$
f([u, v])=[f(u), f(v)], \text { for all } u, v \in \mathfrak{g} .
$$

It is denoted as $\mathfrak{g} \cong \mathfrak{h}$. The lower central series of a Lie algebra $\mathfrak{g}$ is defined as

$$
\mathfrak{g}^{1}=\mathfrak{g}, \mathfrak{g}^{2}=\left[\mathfrak{g}^{1}, \mathfrak{g}\right], \ldots, \mathfrak{g}^{k}=\left[\mathfrak{g}^{k-1}, \mathfrak{g}\right], \ldots
$$

If there exists $m \in \mathbb{N}$ such that $\mathfrak{g}^{m} \equiv 0$, then $\mathfrak{g}$ is called nilpotent. The nilpotency class of $\mathfrak{g}$ if the smallest natural $c$ such that $\mathfrak{g}^{c+1} \equiv 0$.

An $n$-dimensional nilpotent Lie algebra $\mathfrak{g}$ is said to be filiform if it is verified that

$$
\operatorname{dim} \mathfrak{g}^{k}=n-k, \text { for all } k \in\{2, \ldots, n\} .
$$

The only $n$-dimensional filiform Lie algebra for $n<3$ is the abelian. For $n \geq 3$, it is always possible to find an adapted basis $\left\{e_{1}, \ldots, e_{n}\right\}$ of $\mathfrak{g}$ such that

$$
\left\{\begin{array}{l}
{\left[e_{1}, e_{2}\right]=0,} \\
{\left[e_{1}, e_{j}\right]=e_{j-1}, \text { for all } j \in\{3, \ldots, n\},} \\
{\left[e_{2}, e_{j}\right]=\left[e_{3}, e_{j}\right]=0, \text { for all } j \in\{3, \ldots, n\} .}
\end{array}\right.
$$


If $n \geq 4$, then the following two integers are invariants by isomorphism [5].

$$
z_{1}=\min \left\{i \geq 4 \mid\left[e_{i}, e_{n}\right] \neq 0\right\}, \quad z_{2}=\min \left\{i \geq 4 \mid\left[e_{i}, e_{i+1}\right] \neq 0\right\} .
$$

From the condition of filiformity and the Jacobi identity (2.1), the bracket product of $\mathfrak{g}$ is determined by (2.5) and the products

$$
\left[e_{i}, e_{j}\right]=\sum_{k=2}^{\min \{i-1, n-2\}} c_{i j}^{k} e_{k}, \quad \text { for } \quad 4 \leq i<j \leq n,
$$

where $c_{i, j}^{k} \in K$ are called structure constants of $\mathfrak{g}$. If all of them are zeros, then the filiform Lie algebra $\mathfrak{g}$ is called model. From the invariants (2.6), the model algebra is not isomorphic to any other algebra of the same dimension. From (2.5) and (2.7), every $n$-dimensional filiform Lie algebra $\mathfrak{g}$ having an adapted basis $\left\{e_{1}, \ldots, e_{n}\right\}$ verifies that

$$
\mathfrak{g}^{2}=\left\langle e_{2}, \ldots, e_{n-1}\right\rangle, \mathfrak{g}^{3}=\left\langle e_{2}, \ldots, e_{n-2}\right\rangle, \ldots, \mathfrak{g}^{n-1}=\left\langle e_{2}\right\rangle, \mathfrak{g}^{n}=0 .
$$

\subsection{Invariant functions in Lie algebras}

In this subsection we recall the definitions and main properties of invariant functions $\psi$ and $\varphi$, obtained by Hrivnák and Novotný [17] in 2007.

\subsubsection{The invariant function $\psi$}

Definition 2.1. Let $\mathfrak{g}$ be a Lie algebra. An endomorphism $d$ of $\mathfrak{g}$ is said to be a $(\alpha, \beta, \gamma)$-derivation of $\mathfrak{g}$ if there exist $\alpha, \beta, \gamma \in \mathbb{C}$ such that

$$
\alpha d[X, Y]=\beta[d X, Y]+\gamma[X, d Y], \quad \forall X, Y \in \mathfrak{g} .
$$

The set of $(\alpha, \beta, \gamma)$-derivations of $\mathfrak{g}$ will be denoted by $\operatorname{Der}_{(\alpha, \beta, \gamma)} \mathfrak{g}$.

Note that this definition is the extension of the usual definition of derivation, that is, $d[X, Y]=$ $[d X, Y]+[X, d Y], \forall X, Y \in \mathfrak{g}$.

Theorem 2.1. Let $f: \mathfrak{g} \mapsto \widetilde{\mathfrak{g}}$ be an isomorphism between two complex Lie algebras $\mathfrak{g}$ and $\widetilde{\mathfrak{g}}$. Then, the mapping $\rho:$ End $\mathfrak{g} \mapsto E n d \widetilde{\mathfrak{g}}$ defined as $\rho(d)=f d f^{-1}$ is an isomorphism between the corresponding vector spaces $\operatorname{Der}_{(\alpha, \beta, \gamma)} \mathfrak{g}$ and $\operatorname{Der}_{(\alpha, \beta, \gamma)} \widetilde{\mathfrak{g}}, \forall \alpha, \beta, \gamma \in \mathbb{C}$.

Corollary 2.1. The dimension of the vector space $\operatorname{Der}_{(\alpha, \beta, \gamma)} \mathfrak{g}$ is an invariant of the Lie algebra $\mathfrak{g}$, $\forall \alpha, \beta, \gamma \in \mathbb{C}$.

Definition 2.2. The functions $\psi_{\mathfrak{g}}, \psi_{\mathfrak{g}}^{0}: \mathbb{C} \mapsto\left\{0,1,2, \ldots,(\operatorname{dim} \mathfrak{g})^{2}\right\}$ defined as

$$
\begin{aligned}
& \left(\psi_{\mathfrak{g}}\right)(\alpha)=\operatorname{dim} \operatorname{Der}_{(\alpha, 1,1)} \mathfrak{g}, \\
& \left(\psi_{\mathfrak{g}}^{0}\right)(\alpha)=\operatorname{dim} \operatorname{Der}_{(\alpha, 1,0)} \mathfrak{g},
\end{aligned}
$$

are called $\psi_{\mathfrak{g}}$ and $\psi_{\mathfrak{g}}^{0}$ invariant functions corresponding to the $(\alpha, \beta, \gamma)$-derivations of $\mathfrak{g}$.

A relevant result obtained by Hrivnák and Novotný [17] is the following: 
Theorem 2.2. Two 3-dimensional complex Lie algebras $\mathfrak{g}_{1}$ and $\mathfrak{g}_{2}$ are isomorphic if and only if $\psi_{\mathfrak{g}_{1}}=\psi_{\mathfrak{g}_{2}}$.

A sketch of the proof of this result, which is given in a complete form in [12] is the following: Hrivnák proves in the first place that if $f: A \mapsto \tilde{A}$ is an isomorphism of complex algebras $A$ and $\tilde{A}$, then the mapping $\rho$ : End $A \mapsto$ End $\amalg \tilde{A}$ is an isomorphism of the vector spaces $\operatorname{der}_{(\alpha, \beta, \gamma)} A$ and $\operatorname{der}_{(\alpha, \beta, \gamma)} \tilde{A}$, being the mapping $\rho$ defined for all $D \in$ End $A$ by $\rho(D)=f D f^{-1}$. Then, as a consequence of this result is that for any $(\alpha, \beta, \gamma) \in \mathbb{C}$, the dimension of the vector space $\operatorname{der}_{(\alpha, \beta, \gamma)} A$ is an invariant characteristic of algebras. From this last result the previous theorem is deduced with a different notation.

\subsubsection{The invariant function $\varphi$}

Definition 2.3. Let $(V, f)$ be a representation of the Lie algebra $\mathfrak{g}$, where $V$ is a complex vector space. A $V$-cochain of dimension $q$ is a $q$-linear mapping

$$
c: \underbrace{\mathfrak{g} \times \cdots \times \mathfrak{g}}_{q \text {-times }} \mapsto V
$$

such that $c\left(x_{1}, \ldots, x_{i}, \ldots, x_{j}, \ldots, x_{q}\right)+c\left(x_{1}, \ldots, x_{j}, \ldots, x_{i}, \ldots, x_{q}\right)=0$, for all indices $i, j$, with $1 \leq i<$ $j \leq q$.

The vector space of all $V$-cochains of dimension $q$ with $q \in \mathbb{N}$ and $C^{0}(\mathfrak{g}, V)=V$ will be denoted by $C^{q}(\mathfrak{g}, V)$. Now, we define the mapping $d: C^{q}(\mathfrak{g}, V) \mapsto C^{q+1}(\mathfrak{g}, V)$, with $q=0,1,2, \ldots$ as

$$
\begin{aligned}
d c(x) & =f(x) c, \text { with } c \in C^{0}(\mathfrak{g}, V), \\
d c\left(x_{1}, \ldots, x_{q+1}\right) & =\sum_{i=1}^{q+1}(-1)^{i+1} f\left(x_{i}\right) c\left(x_{1}, \ldots, \widehat{x_{i}}, \ldots, x_{q+1}\right) \\
& +\sum_{\substack{i, j=1 \\
i<j}}^{q+1}(-1)^{i+j} c\left(\left[x_{i}, x_{j}\right], x_{1}, \ldots, \widehat{x_{i}}, \ldots, \widehat{x_{j}}, \ldots, x_{q+1}\right),
\end{aligned}
$$

where $\widehat{x_{i}}$ means that $x_{i}$ has been omitted.

Under the same conditions as before, let $\kappa=\left(\kappa_{i j}\right)$ be a symmetric complex matrix of dimension $(q+1) \times(q+1)$.

Definition 2.4. A $\kappa$-twisted cocycle (or simply $\kappa$-cocycle) is any $c \in C^{q}(\mathfrak{g}, V)$, with $q \in \mathbb{N}$, verifying

$$
\begin{aligned}
0 & =\sum_{i=1}^{q+1}(-1)^{i+1} \kappa_{i i} f\left(x_{i}\right) c\left(x_{1}, \ldots, \widehat{x_{i}}, \ldots, x_{q+1}\right) \\
& +\sum_{\substack{i, j=1 \\
i<j}}^{q+1}(-1)^{i+j} \kappa_{i j} c\left(\left[x_{i}, x_{j}\right], x_{1}, \ldots, \widehat{x}_{i}, \ldots, \widehat{x_{j}}, \ldots, x_{q+1}\right) .
\end{aligned}
$$

If the vector space $V$ is identified with the algebra $\mathfrak{g}$, then the adjoint representation can be used as an action (see [8]). So, the equality of the definition 2.4 can be written as 


$$
\begin{aligned}
0 & =\sum_{i=1}^{q+1}(-1)^{i+1} \kappa_{i i}\left[x_{i}, c\left(x_{1}, \ldots, \widehat{x_{i}}, \ldots, x_{q+1}\right)\right] \\
& +\sum_{\substack{i, j=1 \\
i<j}}^{q+1}(-1)^{i+j} \kappa_{i j} c\left(\left[x_{i}, x_{j}\right], x_{1}, \ldots, \widehat{x}_{i}, \ldots, \widehat{x_{j}}, \ldots, x_{q+1}\right) .
\end{aligned}
$$

The set of all $\kappa$-cocycles of dimension $q$ will be denoted by $Z^{q}(\mathfrak{g}, f, \kappa)$. Clearly, it is a vector subspace of $C^{q}(\mathfrak{g}, V)$.

If the following notation is considered

$$
\operatorname{coc}\left(\alpha_{1}, \alpha_{2}, \alpha_{3}, \beta_{1}, \beta_{2}, \beta_{3}\right)=Z^{2}\left(\mathfrak{g}, a d_{\mathfrak{g}},\left(\begin{array}{lll}
\beta_{1} & \alpha_{2} & \alpha_{3} \\
\alpha_{2} & \beta_{3} & \alpha_{1} \\
\alpha_{3} & \alpha_{1} & \beta_{2}
\end{array}\right)\right)
$$

it is easy to see that the vector space $\operatorname{coc}\left(\alpha_{1}, \alpha_{2}, \alpha_{3}, \beta_{1}, \beta_{2}, \beta_{3}\right)$ is constituted by the $B \in C^{2}(\mathfrak{g}, \mathfrak{g})$ such that $\forall X, Y, Z \in \mathfrak{g}$,

$$
\begin{aligned}
0 & =\alpha_{1} B(X,[Y, Z])+\alpha_{2} B(Z,[X, Y])+\alpha_{3} B(Y,[Z, X]) \\
& +\beta_{1}[X, B(Y, Z)]+\beta_{2}[Z, B(X, Y)]+\beta_{3}[Y, B(Z, X)] .
\end{aligned}
$$

Theorem 2.3. Let $g: \mathfrak{g} \mapsto \widetilde{\mathfrak{g}}$ be an isomorphism between Lie algebras $\mathfrak{g}$ and $\widetilde{\mathfrak{g}}$. Then, the mapping $\rho: C^{q}(\mathfrak{g}, \mathfrak{g}) \mapsto C^{q}(\widetilde{\mathfrak{g}}, \widetilde{\mathfrak{g}})$, for $q \in \mathbb{N}$, defined by $(\rho c)\left(x_{1}, \ldots, x_{q}\right)=g c\left(g^{-1} x_{1}, \ldots, g^{-1} x_{q}\right), \forall c \in C^{q}(\mathfrak{g}, \mathfrak{g})$ and $\forall x_{1}, \ldots, x_{q} \in \widetilde{\mathfrak{g}}$, is an isomorphism between the vector spaces $C^{q}(\mathfrak{g}, \mathfrak{g})$ and $C^{q}(\widetilde{\mathfrak{g}}, \widetilde{\mathfrak{g}})$.

Corollary 2.2. The dimension of the vector space $Z^{q}\left(\mathfrak{g}, a d_{\mathfrak{g}}, \kappa\right)$ is an invariant of the Lie algebra $\mathfrak{g}$, for any $q \in \mathbb{N}$ and any complex $(q+1)$-square symmetric matrix $\kappa$.

Definition 2.5. The invariant functions $\varphi$ and $\varphi^{0}$ corresponding to the $n$-dimensional Lie algebra $\mathfrak{g}$ are defined as

$$
\begin{gathered}
\varphi: \mathbb{C} \mapsto\left\{0,1, \ldots, \frac{n^{2}(n-1)}{2}\right\} \\
(\varphi \mathfrak{g})(\alpha)=\operatorname{dim} \operatorname{coc}_{(1,1,1, \alpha, \alpha, \alpha)} \mathfrak{g}
\end{gathered}
$$

and

$$
\begin{aligned}
& \varphi^{0}: \mathbb{C} \mapsto\left\{0,1, \ldots, \frac{n^{2}(n-1)}{2}\right\} \\
& \left(\varphi^{0} \mathfrak{g}\right)(\alpha)=\operatorname{dim} \operatorname{coc}_{(0,1,1, \alpha, 1,1)} \mathfrak{g},
\end{aligned}
$$

respectively. 


\subsection{Proper contractions of Lie algebras}

Let $\mathfrak{g}=(V,[]$,$) be an n$-dimensional Lie algebra and $U:(0,1] \mapsto \mathfrak{g l}(V)$ be an one-parameter mapping. If the limit

$$
[X, Y]_{0}=\lim _{\varepsilon \rightarrow 0^{+}} U^{-1}(\varepsilon)[U(\varepsilon) X, U(\varepsilon) Y]
$$

exists for all $X, Y \in \mathfrak{g}$, we say that $\mathfrak{g}_{0}=\left(V,[,]_{0}\right)$ is an one-parameter contraction of the algebra $\mathfrak{g}$ and we write $\mathfrak{g} \mapsto \mathfrak{g}_{0}$. The contraction $\mathfrak{g} \mapsto \mathfrak{g}_{0}$ is said to be proper if $\mathfrak{g}$ is not isomorphic to $\mathfrak{g}_{0}$.

The following results were shown in [18].

Theorem 2.4. If $\mathfrak{g}_{0}$ is a proper contraction of the complex Lie algebra $\mathfrak{g}$, then

1. $\operatorname{dim} \operatorname{Der}(\mathfrak{g})<\operatorname{dim} \operatorname{Der}\left(\mathfrak{g}_{0}\right)$.

2. $\psi \mathfrak{g} \leq \psi \mathfrak{g}_{0}$ and $\psi \mathfrak{g}(1)<\psi \mathfrak{g}_{0}(1)$.

3. $\varphi \mathfrak{g} \leq \varphi \mathfrak{g}_{0}$ and $\varphi^{0} \mathfrak{g} \leq \varphi^{0} \mathfrak{g}_{0}$,

where $\psi$ and $\varphi$ are the invariant functions introduced by Hrivnák and Novotný in [17].

Moreover, it is satisfied that, in dimension 3, Condition 2 is a characterization of proper contractions of $\mathfrak{g}$.

\section{The $\psi$ and $\varphi$ invariant functions for 3-dimensional filiform Lie algebras}

In this and next sections we extend the definitions of the invariant functions $\psi$ and $\varphi$ introduced by Hrivnák and Novotný [17] to the case of filiform Lie algebras of lower dimensions. We study, in the first place, the case of dimension 3.

\subsection{The $\psi$ invariant function for 3-dimensional filiform Lie algebras}

Let $\mathfrak{f}_{3}$ be the filiform Lie algebra of dimension 3 defined by the law $\left[e_{1}, e_{3}\right]=e_{2}$ (remember that, by agreement, all possible brackets not appearing in the expression of the law are considered null, that is, in this case, $\left.\left[e_{1}, e_{2}\right]=\left[e_{2}, e_{3}\right]=0\right)$.

Let us consider $d \in \operatorname{Der}_{(\alpha, 1,1)} \mathfrak{f}_{3}$. Then

$$
\alpha d([X, Y])=[d(X), Y]+[X, d(Y)] \quad \forall X, Y \in \mathfrak{f}_{3} .
$$

Also, let

$$
\left(\begin{array}{lll}
a_{11} & a_{12} & a_{13} \\
a_{21} & a_{22} & a_{23} \\
a_{31} & a_{32} & a_{33}
\end{array}\right)
$$

be the matrix associated with the endomorphism $d$.

We wish to obtain the elements of this matrix. To do this, for the pair of generators $\left(e_{1}, e_{2}\right)$ the condition (3.1) is now

$$
\alpha d\left(\left[e_{1}, e_{2}\right]\right)=\left[d\left(e_{1}\right), e_{2}\right]+\left[e_{1}, d\left(e_{2}\right)\right],
$$

and $d\left(e_{i}\right)=\sum_{h=1}^{3} a_{i h} e_{h}$. We can get the first condition to be fulfilled for the elements of this endomorphism according to 


$$
\begin{gathered}
\alpha d\left(\left[e_{1}, e_{2}\right]\right)=\alpha d(0)=0 \\
=\left[a_{11} e_{1}+a_{12} e_{2}+a_{13} e_{3}, e_{2}\right]+\left[e_{1}, a_{21} e_{1}+a_{22} e_{2}+a_{23} e_{3}\right]=a_{23} e_{2},
\end{gathered}
$$

which implies $a_{23}=0$.

Proceeding in the same way with the following pair $\left(e_{1}, e_{3}\right)$, we have that from $\alpha d\left(\left[e_{1}, e_{3}\right]\right)=$ $\left[d\left(e_{1}\right), e_{3}\right]+\left[e_{1}, d\left(e_{3}\right)\right]$, we deduce, by taking into account the law of the algebra, that

$$
\alpha d\left(e_{2}\right)=\left[a_{11} e_{1}+a_{12} e_{2}+a_{13} e_{3}, e_{3}\right]+\left[e_{1}, a_{31} e_{1}+a_{32} e_{2}+a_{33} e_{3}\right]=\left(a_{11}+a_{33}\right) e_{2} .
$$

So, $\alpha a_{21} e_{1}+\alpha a_{22} e_{2}+\alpha a_{23} e_{3}=\left(a_{11}+a_{33}\right) e_{2}$, and thus, according to the linear dependence, the following conditions are obtained

$$
\alpha a_{21}=0, \quad \alpha a_{22}=a_{11}+a_{33}, \quad \alpha a_{23}=0
$$

Finally, by proceeding in the same way with the last pair $\left(e_{2}, e_{3}\right)$, we have that

$$
\begin{aligned}
\alpha d\left(\left[e_{2}, e_{3}\right]\right) & =\left[d\left(e_{2}\right), e_{3}\right]+\left[e_{2}, d\left(e_{3}\right)\right] \equiv \\
0=\left[a_{21} e_{1}+a_{22} e_{2}+a_{23} e_{3}, e_{3}\right] & +\left[e_{2}, a_{31} e_{1}+a_{32} e_{2}+a_{33} e_{3}\right]=a_{21} e_{2}=0 .
\end{aligned}
$$

As a result, we find that $a_{21}=0$.

Summarizing, we have obtained the following conditions:

\begin{tabular}{|c|l|}
\hline From pair $\left(e_{i}, e_{j}\right)$ & Conditions \\
\hline \hline$\left(e_{1}, e_{2}\right)$ & $a_{23}=0$. \\
\hline$\left(e_{1}, e_{3}\right)$ & $\alpha a_{21}=0, \quad \alpha a_{22}=a_{11}+a_{33}, \quad \alpha a_{23}=0$. \\
\hline$\left(e_{2}, e_{3}\right)$ & $a_{21}=0$. \\
\hline
\end{tabular}

which allow us to determine the vector space $\operatorname{Der}_{(\alpha, 1,1)} \mathfrak{f}_{3}$. Indeed, we have that: for $\alpha \neq 0$ :

$$
\begin{aligned}
\operatorname{Der}_{(\alpha, 1,1)} \mathfrak{f}_{3}=\operatorname{span}_{\mathbb{C}}\{ & \left(\begin{array}{ccc}
\alpha & 0 & 0 \\
0 & 1 & 0 \\
0 & 0 & 0
\end{array}\right),\left(\begin{array}{ccc}
-1 & 0 & 0 \\
0 & 0 & 0 \\
0 & 0 & 1
\end{array}\right),\left(\begin{array}{lll}
0 & 1 & 0 \\
0 & 0 & 0 \\
0 & 0 & 0
\end{array}\right), \\
& \left.\left(\begin{array}{lll}
0 & 0 & 1 \\
0 & 0 & 0 \\
0 & 0 & 0
\end{array}\right),\left(\begin{array}{lll}
0 & 0 & 0 \\
0 & 0 & 0 \\
1 & 0 & 0
\end{array}\right),\left(\begin{array}{lll}
0 & 0 & 0 \\
0 & 0 & 0 \\
0 & 1 & 0
\end{array}\right)\right\} ;
\end{aligned}
$$

for $\alpha=0$ :

$$
\begin{aligned}
\operatorname{Der}_{(0,1,1)} \mathfrak{f}_{3}=\operatorname{span}_{\mathbb{C}}\{ & \left(\begin{array}{ccc}
-1 & 0 & 0 \\
0 & 0 & 0 \\
0 & 0 & 1
\end{array}\right),\left(\begin{array}{lll}
0 & 1 & 0 \\
0 & 0 & 0 \\
0 & 0 & 0
\end{array}\right),\left(\begin{array}{lll}
0 & 0 & 1 \\
0 & 0 & 0 \\
0 & 0 & 0
\end{array}\right), \\
& \left.\left(\begin{array}{lll}
0 & 0 & 0 \\
0 & 1 & 0 \\
0 & 0 & 0
\end{array}\right),\left(\begin{array}{lll}
0 & 0 & 0 \\
0 & 0 & 0 \\
1 & 0 & 0
\end{array}\right),\left(\begin{array}{lll}
0 & 0 & 0 \\
0 & 0 & 0 \\
0 & 1 & 0
\end{array}\right)\right\} .
\end{aligned}
$$


Therefore, $\psi_{\mathfrak{f}_{3}(\alpha)}=6, \forall \alpha \in \mathbb{C}$. According to the notation used in [17], it is expressed in the following way

\begin{tabular}{|c||c|}
\hline$\alpha$ & $\forall \alpha \in \mathbb{C}$ \\
\hline$\psi_{\mathfrak{f}_{3}(\alpha)}$ & 6 \\
\hline
\end{tabular}

Note that this dimension is always the same independently of the value of $\alpha$.

\subsection{The $\varphi$ invariant function for 3-dimensional filiform Lie algebras}

The $\mathfrak{f}_{3}$-cochains $B_{i} \in C^{2}\left(\mathfrak{f}_{3}, \mathfrak{f}_{3}\right), \forall i \in\left\{0,1, \ldots, \frac{n^{2}(n-1)}{2}\right\}$, are defined starting from their non-null commutativity relations $B_{r}\left(e_{s}, e_{t}\right)=k e_{u}, s<t$ which verify the equality Eq. (2.18). The $\mathfrak{f}_{3}$-cochains which constitute a basis of the vector space $\operatorname{coc}_{(1,1,1, \lambda, \lambda, \lambda)} \mathfrak{f}_{3}$ whose dimension determines the invariant function $\varphi_{\mathfrak{f}_{3}}$ are the following:

For $\lambda=0$ :

$$
\begin{array}{lll}
B_{1}: B_{1}\left(e_{1}, e_{2}\right)=e_{1} & B_{2}: B_{2}\left(e_{1}, e_{2}\right)=e_{2} & B_{3}: B_{3}\left(e_{1}, e_{2}\right)=e_{3} \\
B_{4}: B_{4}\left(e_{2}, e_{3}\right)=e_{1} & B_{5}: B_{5}\left(e_{2}, e_{3}\right)=e_{2} & B_{6}: B_{6}\left(e_{2}, e_{3}\right)=e_{3} \\
B_{7}: B_{7}\left(e_{1}, e_{3}\right)=e_{1} & B_{8}: B_{8}\left(e_{1}, e_{3}\right)=e_{2} & B_{9}: B_{9}\left(e_{1}, e_{3}\right)=e_{3}
\end{array}
$$

For $\lambda \neq 0$ :

$$
\begin{array}{lll}
C_{1}: C_{1}\left(e_{1}, e_{2}\right)=e_{1}, & C_{1}\left(e_{2}, e_{3}\right)=e_{3} & C_{2}: C_{2}\left(e_{2}, e_{3}\right)=e_{2} \\
C_{3}: C_{3}\left(e_{2}, e_{3}\right)=e_{1} & & C_{4}: C_{4}\left(e_{1}, e_{2}\right)=e_{2} \\
C_{5}: C_{5}\left(e_{1}, e_{2}\right)=e_{3} & & C_{6}: C_{6}\left(e_{1}, e_{3}\right)=e_{1} \\
C_{7}: C_{7}\left(e_{1}, e_{3}\right)=e_{2} & & C_{8}: C_{8}\left(e_{1}, e_{3}\right)=e_{3}
\end{array}
$$

Therefore, we have the following vector spaces

$$
\begin{aligned}
& \operatorname{coc}_{(1,1,1,0,0,0)} \mathfrak{f}_{3}=\operatorname{span}_{\mathbb{C}}\left\{B_{i}, 1 \leq i \leq 9\right\} \\
& \operatorname{coc}_{(1,1,1, \lambda, \lambda, \lambda)} \mathfrak{f}_{3}=\operatorname{span}_{\mathbb{C}}\left\{C_{i}, 1 \leq i \leq 8\right\}, \forall \lambda \neq 0 .
\end{aligned}
$$

So, we have as a result

\begin{tabular}{|c||c|c|}
\hline$\lambda$ & 0 & $\forall \lambda \in \mathbb{C} \backslash\{0\}$ \\
\hline$\varphi_{\mathfrak{f}_{3}}(\lambda)$ & 9 & 8 \\
\hline
\end{tabular}

Let us now consider these computations for 4-dimensional filiform Lie algebras.

\section{The $\psi$ and $\varphi$ invariant functions for 4-dimensional filiform Lie algebras}

In this section we deal with the case of dimension 4 with the purpose of comparing the obtained results with previous ones. 


\subsection{The $\psi$ invariant function for 4-dimensional filiform Lie algebras}

Let $\mathfrak{f}_{4}$ be a filiform Lie algebra of dimension 4 defined by the brackets $\left[e_{1}, e_{3}\right]=e_{2}$ and $\left[e_{1}, e_{4}\right]=e_{3}$.

In the same way as before, we wish to obtain a basis of the vector space $\operatorname{Der}_{(\alpha, 1,1)} \mathfrak{f}_{4}, \forall \alpha \in \mathbb{C}$. To do this, let us consider $d \in \operatorname{Der}_{(\alpha, 1,1)} \mathfrak{f}_{4}$. Then

$$
\alpha d([X, Y])=[d(X), Y]+[X, d(Y)] \quad \forall X, Y \in \mathfrak{f}_{4} .
$$

Let

$$
\left(\begin{array}{llll}
a_{11} & a_{12} & a_{13} & a_{14} \\
a_{21} & a_{22} & a_{23} & a_{24} \\
a_{31} & a_{32} & a_{33} & a_{34} \\
a_{41} & a_{42} & a_{43} & a_{44}
\end{array}\right)
$$

be the associated matrix with the endomorphism $d$. As

$$
\alpha d([X, Y])=[d(X), Y]+[X, d(Y)] \quad \forall X, Y \in \mathfrak{f}_{4}
$$

we have, in the first place, that $\alpha d\left(\left[e_{1}, e_{2}\right]\right)=\left[d\left(e_{1}\right), e_{2}\right]+\left[e_{1}, d\left(e_{2}\right)\right]$, which allows us to obtain the first condition for the elements of the previous matrix. As $\left[e_{1}, a_{21} e_{1}+a_{22} e_{2}+a_{23} e_{3}+a_{24} e_{4}\right]=0$, it implies that $a_{23} e_{2}+a_{24} e_{3}=0$, and so, $a_{23}=a_{24}=0$.

Similarly, we obtain the rest of conditions for the elements of the matrix in the same way as we did in Subsection 3.1. These conditions will allow us to obtain the vector space $\operatorname{Der}_{(\alpha, 1,1)} \mathfrak{f}_{4}$.

\begin{tabular}{|c|l|}
\hline From pair $\left(e_{i}, e_{j}\right)$ & Conditions \\
\hline \hline$\left(e_{1}, e_{2}\right)$ & $a_{24}=0, \quad a_{23}=0$. \\
\hline$\left(e_{1}, e_{3}\right)$ & $\alpha a_{21}=0, \quad \alpha a_{22}=a_{11}+a_{33}$, \\
& $\alpha a_{23}=a_{34}, \quad \alpha a_{24}=0$. \\
\hline$\left(e_{1}, e_{4}\right)$ & $\alpha a_{31}=0, \quad \alpha a_{32}=a_{43}$, \\
& $\alpha a_{33}=a_{11}+a_{44}, \quad \alpha a_{34}=0$. \\
\hline$\left(e_{2}, e_{3}\right)$ & $a_{21}=0$. \\
\hline$\left(e_{3}, e_{4}\right)$ & $a_{31}=a_{41}=0$. \\
\hline
\end{tabular}

Note that no conditions are obtained from some pairs of generators, for instance, from the pair $\left(e_{2}, e_{4}\right)$ in this case.

Now, starting from these results we obtain the vector space $\operatorname{Der}_{(\alpha, 1,1)} \mathfrak{f}_{4}$, being $\alpha \neq 0$, and $\operatorname{Der}_{(0,1,1)} \mathfrak{f}_{4}$.

For $\alpha \neq 0$ :

$$
\begin{gathered}
\operatorname{Der}_{(\alpha, 1,1)} \mathfrak{f}_{4}=\operatorname{span}_{\mathbb{C}}\left\{\left(\begin{array}{cccc}
1 & 0 & 0 & 0 \\
0 & \frac{1}{\alpha} & 0 & 0 \\
0 & 0 & 0 & 0 \\
0 & 0 & 0 & -1
\end{array}\right),\left(\begin{array}{llll}
0 & 1 & 0 & 0 \\
0 & 0 & 0 & 0 \\
0 & 0 & 0 & 0 \\
0 & 0 & 0 & 0
\end{array}\right),\left(\begin{array}{llll}
0 & 0 & 1 & 0 \\
0 & 0 & 0 & 0 \\
0 & 0 & 0 & 0 \\
0 & 0 & 0 & 0
\end{array}\right),\right. \\
\left.\left(\begin{array}{llll}
0 & 0 & 0 & 1 \\
0 & 0 & 0 & 0 \\
0 & 0 & 0 & 0 \\
0 & 0 & 0 & 0
\end{array}\right),\left(\begin{array}{llll}
0 & 0 & 0 & 0 \\
0 & 0 & 0 & 0 \\
0 & 1 & 0 & 0 \\
0 & 0 & \alpha & 0
\end{array}\right),\left(\begin{array}{llll}
0 & 0 & 0 & 0 \\
0 & 0 & 0 & 0 \\
0 & 0 & 0 & 0 \\
0 & 1 & 0 & 0
\end{array}\right),\left(\begin{array}{llll}
0 & 0 & 0 & 1 \\
0 & \frac{1}{\alpha} & 0 & 0 \\
0 & 0 & 1 & 0 \\
0 & 0 & 0 & \alpha
\end{array}\right)\right\} .
\end{gathered}
$$


For $\alpha=0$ :

$$
\begin{gathered}
\operatorname{Der}_{(0,1,1)} \mathfrak{f}_{4}=\operatorname{span}_{\mathbb{C}}\left\{\left(\begin{array}{cccc}
1 & 0 & 0 & 0 \\
0 & 0 & 0 & 0 \\
0 & 0 & -1 & 0 \\
0 & 0 & 0 & -1
\end{array}\right),\left(\begin{array}{llll}
0 & 1 & 0 & 0 \\
0 & 0 & 0 & 0 \\
0 & 0 & 0 & 0 \\
0 & 0 & 0 & 0
\end{array}\right),\left(\begin{array}{llll}
0 & 0 & 1 & 0 \\
0 & 0 & 0 & 0 \\
0 & 0 & 0 & 0 \\
0 & 0 & 0 & 0
\end{array}\right),\right. \\
\left.\left(\begin{array}{llll}
0 & 0 & 0 & 1 \\
0 & 0 & 0 & 0 \\
0 & 0 & 0 & 0 \\
0 & 0 & 0 & 0
\end{array}\right),\left(\begin{array}{llll}
0 & 0 & 0 & 0 \\
0 & 1 & 0 & 0 \\
0 & 0 & 0 & 0 \\
0 & 0 & 0 & 0
\end{array}\right),\left(\begin{array}{llll}
0 & 0 & 0 & 0 \\
0 & 0 & 0 & 0 \\
0 & 1 & 0 & 0 \\
0 & 0 & 0 & 0
\end{array}\right),\left(\begin{array}{llll}
0 & 0 & 0 & 0 \\
0 & 0 & 0 & 0 \\
0 & 0 & 0 & 0 \\
0 & 1 & 0 & 0
\end{array}\right)\right\} .
\end{gathered}
$$

So we conclude that

\begin{tabular}{|c||c|}
\hline$\alpha$ & $\forall \alpha \in \mathbb{C}$ \\
\hline$\psi_{\mathfrak{f}_{4}}(\alpha)$ & 7 \\
\hline
\end{tabular}

\subsection{The $\varphi$ invariant function for 4-dimensional filiform Lie algebras}

As we see in the case of dimension 3 , the $\mathfrak{f}_{4}$-cochains $B_{i} \in C^{2}\left(\mathfrak{f}_{4}, \mathfrak{f}_{4}\right), \forall i \in\left\{0,1, \ldots, \frac{n^{2}(n-1)}{2}\right\}$ are defined starting from their non-null commutativity relations $B_{r}\left(e_{s}, e_{t}\right)=k e_{u}, s<t$, which verify the equality given by Eq. (2.18). The $\mathfrak{f}_{4}$-cochains which constitute a basis of the vector space $\operatorname{coc}_{(1,1,1, \lambda, \lambda, \lambda)} \mathfrak{f}_{4}$, whose dimension determines the invariant function $\varphi_{\mathfrak{f}_{4}}$, are the following:

For $\lambda=0$ :

\begin{tabular}{ll|ll|ll}
$B_{1}:$ & $B_{1}\left(e_{1}, e_{2}\right)=e_{1}$. & $B_{2}:$ & $B_{2}\left(e_{1}, e_{2}\right)=e_{2}$. & $B_{3}:$ & $B_{3}\left(e_{1}, e_{2}\right)=e_{3}$. \\
$B_{4}:$ & $B_{4}\left(e_{1}, e_{2}\right)=e_{4}$. & $B_{5}:$ & $B_{5}\left(e_{1}, e_{4}\right)=e_{1}$. & $B_{6}:$ & $B_{6}\left(e_{1}, e_{4}\right)=e_{2}$. \\
$B_{7}:$ & $B_{7}\left(e_{1}, e_{4}\right)=e_{3}$. & $B_{8}:$ & $B_{8}\left(e_{1}, e_{4}\right)=e_{4}$. & $B_{9}:$ & $B_{9}\left(e_{1}, e_{3}\right)=e_{1}$. \\
$B_{10}:$ & $B_{10}\left(e_{1}, e_{3}\right)=e_{2}$. & $B_{11}:$ & $B_{11}\left(e_{1}, e_{3}\right)=e_{3}$. & $B_{12}:$ & $B_{12}\left(e_{1}, e_{3}\right)=e_{4}$. \\
$B_{13}:$ & $B_{13}\left(e_{1}, e_{4}\right)=e_{1}$. & $B_{14}:$ & $B_{14}\left(e_{1}, e_{4}\right)=e_{2}$. & $B_{15}:$ & $B_{15}\left(e_{1}, e_{4}\right)=e_{3}$. \\
$B_{16}:$ & $B_{16}\left(e_{1}, e_{4}\right)=e_{4}$. & & & &
\end{tabular}

For $\lambda \neq 0$ :

$$
\begin{array}{lll}
C_{1}: C_{1}\left(e_{1}, e_{2}\right)=e_{2} . & C_{2}: C_{2}\left(e_{1}, e_{2}\right)=e_{3} . & C_{3}: C_{3}\left(e_{1}, e_{2}\right)=e_{4} . \\
C_{4}: C_{4}\left(e_{1}, e_{4}\right)=e_{2} . & C_{5}: C_{5}\left(e_{1}, e_{4}\right)=e_{3} . & C_{6}: C_{6}\left(e_{1}, e_{4}\right)=e_{4} . \\
C_{7}: C_{7}\left(e_{1}, e_{3}\right)=e_{2} . & C_{8}: C_{8}\left(e_{1}, e_{3}\right)=e_{3} . & C_{9}: C_{9}\left(e_{1}, e_{3}\right)=e_{4} . \\
C_{10}: C_{10}\left(e_{3}, e_{4}\right)=e_{1} . & C_{11}: C_{11}\left(e_{3}, e_{4}\right)=e_{2} . & \\
C_{12}: C_{12}\left(e_{2}, e_{4}\right)=\frac{1}{\lambda} e_{3}, & C_{12}\left(e_{2}, e_{3}\right)=e_{2}, & C_{12}\left(e_{1}, e_{3}\right)=-\frac{1}{\lambda^{2}} e_{1} . \\
C_{13}: C_{13}\left(e_{2}, e_{4}\right)=\frac{1}{\lambda} e_{3}, & C_{13}\left(e_{2}, e_{3}\right)=e_{2}, & \\
C_{13}\left(e_{1}, e_{3}\right)=\left(\frac{-1+\lambda^{2}}{\lambda^{2}}\right) e_{1}, & C_{13}\left(e_{3}, e_{4}\right)=e_{4} . & \\
C_{14}: C_{14}\left(e_{2}, e_{4}\right)=\frac{1}{\lambda} e_{3}, & C_{14}\left(e_{2}, e_{3}\right)=e_{2}, & C_{14}\left(e_{1}, e_{3}\right)=\frac{-1}{\lambda^{2}} e_{1}, \\
C_{14}\left(e_{1}, e_{4}\right)=e_{1}, & C_{14}\left(e_{3}, e_{4}\right)=-e_{3} . & \\
C_{15}: C_{15}\left(e_{2}, e_{4}\right)=\frac{1}{\lambda} e_{3}, & C_{15}\left(e_{2}, e_{3}\right)=e_{2}, & C_{15}\left(e_{3}, e_{4}\right)=\frac{1}{\lambda^{2}} e_{4} .
\end{array}
$$




$$
\begin{array}{rlll}
C_{16}: C_{16}\left(e_{2}, e_{3}\right)=e_{3}, & C_{16}\left(e_{2}, e_{4}\right)=\lambda e_{2}, & C_{16}\left(e_{1}, e_{2}\right)=\frac{-1}{\lambda} e_{1}, \\
C_{16}\left(e_{1}, e_{3}\right)=e_{1}, & C_{16}\left(e_{1}, e_{4}\right)=e_{1}, & C_{16}\left(e_{3}, e_{4}\right)=e_{4} . \\
C_{17}: C_{17}\left(e_{2}, e_{3}\right)=e_{3}, & C_{17}\left(e_{2}, e_{4}\right)=\lambda e_{2}, & C_{17}\left(e_{1}, e_{2}\right)=\frac{-1}{\lambda} e_{1}, \\
C_{17}\left(e_{3}, e_{4}\right)=e_{3} . & & \\
C_{18}: C_{18}\left(e_{2}, e_{3}\right)=e_{3}, & C_{18}\left(e_{2}, e_{4}\right)=\lambda e_{2}, & C_{18}\left(e_{1}, e_{2}\right)=\frac{-1}{\lambda} e_{1}, \\
C_{18}\left(e_{1}, e_{4}\right)=e_{1} . & &
\end{array}
$$

Therefore, we have the following vector spaces

$$
\begin{aligned}
\operatorname{coc}_{(1,1,1,0,0,0)} \mathfrak{f}_{4} & =\operatorname{span}_{\mathbb{C}}\left\{B_{i} \mid 1 \leq i \leq 16\right\}, \\
\operatorname{coc}_{(1,1,1, \lambda, \lambda, \lambda)} \mathfrak{f}_{4} & =\operatorname{span}_{\mathbb{C}}\left\{C_{i} \mid 1 \leq i \leq 18\right\}, \forall \lambda \neq 0 .
\end{aligned}
$$

So, according to the notation in [17], the invariant function $\varphi_{\mathfrak{f}_{4}}$ is

\begin{tabular}{|c||c|c|}
\hline$\lambda$ & 0 & $\forall \lambda \in \mathbb{C} \backslash\{0\}$ \\
\hline$\varphi_{\mathfrak{f}_{4}}(\lambda)$ & 16 & 18 \\
\hline
\end{tabular}

Note that unlike the previous case, these dimensions are now dependent on the value of $\lambda$.

\section{The $\psi$ invariant function for filiform Lie algebras of dimension 5}

With the objective of proving that there is not any proper contraction from a Heisenberg algebra to a 5-dimensional filiform Lie algebra, we are going to obtain the invariant function $\psi \mathfrak{f}_{5}$, where $\mathfrak{f}_{5}$ is the 5-dimensional filiform Lie algebra with non-null brackets $\left[e_{1}, e_{3}\right]=e_{2},\left[e_{1}, e_{4}\right]=e_{3},\left[e_{1}, e_{5}\right]=e_{4}$.

So, as we did before, we wish to obtain a basis of $\operatorname{Der}_{(\alpha, 1,1)} \mathfrak{f}_{5} \forall \alpha \in \mathbb{C}$.

Let us consider $d \in \operatorname{Der}_{(\alpha, 1,1)} \mathfrak{f}_{5}$ and let

$$
\left(\begin{array}{lllll}
a_{11} & a_{12} & a_{13} & a_{14} & a_{15} \\
a_{21} & a_{22} & a_{23} & a_{24} & a_{25} \\
a_{31} & a_{32} & a_{33} & a_{34} & a_{35} \\
a_{41} & a_{42} & a_{43} & a_{44} & a_{45} \\
a_{51} & a_{52} & a_{53} & a_{54} & a_{55}
\end{array}\right)
$$

be the associated matrix with the endomorphism $d$. By using the same procedure as in 3.1 and taking into consideration in each step the precedent results obtained in the previous ones we obtain the following conditions

\begin{tabular}{|c|l|}
\hline From pair $\left(e_{i}, e_{j}\right)$ & Conditions \\
\hline \hline$\left(e_{1}, e_{2}\right)$ & $a_{23}=a_{24}=a_{25}=0$. \\
\hline$\left(e_{1}, e_{3}\right)$ & $\alpha a_{21}=0, \quad \alpha a_{22}=a_{11}+a_{33}$, \\
& $\alpha a_{23}=a_{34}, \quad \alpha a_{24}=a_{35}, \quad \alpha a_{25}=0$ \\
\hline$\left(e_{1}, e_{4}\right)$ & $\alpha a_{31}=0, \quad \alpha a_{32}=a_{43}$, \\
& $\alpha a_{33}=a_{11}+a_{44}, \quad \alpha a_{34}=a_{45}, \quad \alpha a_{35}=0$. \\
\hline$\left(e_{1}, e_{5}\right)$ & $\alpha a_{41}=0, \quad \alpha a_{42}=a_{53}$, \\
& $\alpha a_{43}=a_{54}, \quad \alpha a_{44}=a_{11}+a_{55}, \quad \alpha a_{45}=0$. \\
\hline$\left(e_{2}, e_{3}\right)$ & $a_{21}=0$. \\
\hline$\left(e_{3}, e_{4}\right)$ & $a_{31}=a_{41}=0$. \\
\hline$\left(e_{3}, e_{5}\right)$ & $a_{41}=a_{51}=0$. \\
\hline
\end{tabular}


Now, it is straightforward to obtain the vector spaces $\operatorname{Der}_{(\alpha, 1,1)} \mathfrak{f}_{5}, \forall \alpha \in \mathbb{C} \backslash\{0\}$ and $\operatorname{Der}_{(0,1,1)} \mathfrak{f}_{5}$.

For $\alpha \neq 0$ :

$$
\begin{gathered}
\operatorname{Der}_{(\alpha, 1,1)} f_{5}=\operatorname{span}_{\mathbb{C}}\left\{\left(\begin{array}{ccccc}
1 & 0 & 0 & 0 & 0 \\
0 & \frac{\alpha+1}{\alpha} & 0 & 0 & 0 \\
0 & 0 & \frac{1}{\alpha} & 0 & 0 \\
0 & 0 & 0 & 0 & 0 \\
0 & 0 & 0 & 0 & -1
\end{array}\right),\left(\begin{array}{llllll}
0 & 1 & 0 & 0 & 0 \\
0 & 0 & 0 & 0 & 0 \\
0 & 0 & 0 & 0 & 0 \\
0 & 0 & 0 & 0 & 0 \\
0 & 0 & 0 & 0 & 0
\end{array}\right),\right. \\
\left(\begin{array}{lllll}
0 & 0 & 1 & 0 & 0 \\
0 & 0 & 0 & 0 & 0 \\
0 & 0 & 0 & 0 & 0 \\
0 & 0 & 0 & 0 & 0 \\
0 & 0 & 0 & 0 & 0
\end{array}\right),\left(\begin{array}{llllll}
0 & 0 & 0 & 1 & 0 \\
0 & 0 & 0 & 0 & 0 \\
0 & 0 & 0 & 0 & 0 \\
0 & 0 & 0 & 0 & 0 \\
0 & 0 & 0 & 0 & 0
\end{array}\right),\left(\begin{array}{lllll}
0 & 0 & 0 & 0 & 1 \\
0 & 0 & 0 & 0 & 0 \\
0 & 0 & 0 & 0 & 0 \\
0 & 0 & 0 & 0 & 0 \\
0 & 0 & 0 & 0 & 0
\end{array}\right),\left(\begin{array}{llllll}
0 & 0 & 0 & 0 & 0 \\
0 & \frac{1}{\alpha^{2}} & 0 & 0 & 0 \\
0 & 0 & \frac{1}{\alpha} & 0 & 0 \\
0 & 0 & 0 & 1 & 0 \\
0 & 0 & 0 & 0 & \alpha
\end{array}\right), \\
\left.\left(\begin{array}{lllllll}
0 & 0 & 0 & 0 & 0 \\
0 & 0 & 0 & 0 & 0 \\
0 & 1 & 0 & 0 & 0 \\
0 & 0 & \alpha & 0 & 0 \\
0 & 0 & 0 & \alpha & 0
\end{array}\right),\left(\begin{array}{lllll}
0 & 0 & 0 & 0 & 0 \\
0 & 0 & 0 & 0 & 0 \\
0 & 0 & 0 & 0 & 0 \\
0 & 1 & 0 & 0 & 0 \\
0 & 0 & \alpha & 0 & 0
\end{array}\right),\left(\begin{array}{lllll}
0 & 0 & 0 & 0 & 0 \\
0 & 0 & 0 & 0 & 0 \\
0 & 0 & 0 & 0 & 0 \\
0 & 0 & 0 & 0 & 0 \\
0 & 1 & 0 & 0 & 0
\end{array}\right)\right\},
\end{gathered}
$$

For $\alpha=0$ :

$$
\begin{gathered}
\operatorname{Der}_{(0,1,1)} \mathfrak{f}_{5}=\operatorname{span}_{\mathbb{C}}\left\{\left(\begin{array}{ccccc}
1 & 0 & 0 & 0 & 0 \\
0 & 0 & 0 & 0 & 0 \\
0 & 0 & -1 & 0 & 0 \\
0 & 0 & 0 & -1 & 0 \\
0 & 0 & 0 & 0 & -1
\end{array}\right),\left(\begin{array}{llllll}
0 & 1 & 0 & 0 & 0 \\
0 & 0 & 0 & 0 & 0 \\
0 & 0 & 0 & 0 & 0 \\
0 & 0 & 0 & 0 & 0 \\
0 & 0 & 0 & 0 & 0
\end{array}\right),\right. \\
\left(\begin{array}{lllll}
0 & 0 & 1 & 0 & 0 \\
0 & 0 & 0 & 0 & 0 \\
0 & 0 & 0 & 0 & 0 \\
0 & 0 & 0 & 0 & 0 \\
0 & 0 & 0 & 0 & 0
\end{array}\right),\left(\begin{array}{llllll}
0 & 0 & 0 & 1 & 0 \\
0 & 0 & 0 & 0 & 0 \\
0 & 0 & 0 & 0 & 0 \\
0 & 0 & 0 & 0 & 0 \\
0 & 0 & 0 & 0 & 0
\end{array}\right),\left(\begin{array}{lllll}
0 & 0 & 0 & 0 & 1 \\
0 & 0 & 0 & 0 & 0 \\
0 & 0 & 0 & 0 & 0 \\
0 & 0 & 0 & 0 & 0 \\
0 & 0 & 0 & 0 & 0
\end{array}\right),\left(\begin{array}{llllll}
0 & 0 & 0 & 0 & 0 \\
0 & 1 & 0 & 0 & 0 \\
0 & 0 & 0 & 0 & 0 \\
0 & 0 & 0 & 0 & 0 \\
0 & 0 & 0 & 0 & 0
\end{array}\right), \\
\left.\left(\begin{array}{llllll}
0 & 0 & 0 & 0 & 0 \\
0 & 0 & 0 & 0 & 0 \\
0 & 1 & 0 & 0 & 0 \\
0 & 0 & 0 & 0 & 0 \\
0 & 0 & 0 & 0 & 0
\end{array}\right),\left(\begin{array}{lllll}
0 & 0 & 0 & 0 & 0 \\
0 & 0 & 0 & 0 & 0 \\
0 & 0 & 0 & 0 & 0 \\
0 & 1 & 0 & 0 & 0 \\
0 & 0 & 0 & 0 & 0
\end{array}\right),\left(\begin{array}{lllll}
0 & 0 & 0 & 0 & 0 \\
0 & 0 & 0 & 0 & 0 \\
0 & 0 & 0 & 0 & 0 \\
0 & 0 & 0 & 0 & 0 \\
0 & 1 & 0 & 0 & 0
\end{array}\right)\right\},
\end{gathered}
$$

Therefore,

\begin{tabular}{|c||c|}
\hline$\alpha$ & $\forall \alpha \in \mathbb{C}$ \\
\hline$\psi_{\mathfrak{f}_{5}}(\alpha)$ & 9 \\
\hline
\end{tabular}

\section{Some examples of proper contractions between different types of algebras}

In this section we study two examples of proper contractions from filiform Lie algebras of lower dimensions to different types of algebras. 


\subsection{Proper contractions of 3-dimensional filiform Lie algebras}

Theorem 2.4 allows us to know if there exists a proper contraction between 3-dimensional Lie algebras $\mathfrak{g}_{1}, \mathfrak{g}_{3,1}, \mathfrak{g}_{2,1} \oplus \mathfrak{g}_{1}, \mathfrak{g}_{3,2}, \mathfrak{g}_{3,3}$ and the 3-dimensional filiform Lie Algebras already studied in this paper. The corresponding invariant functions $\psi_{3 \mathfrak{g}_{1}}, \psi_{\mathfrak{g}_{3,1}}, \psi_{\mathfrak{g}_{2,1} \oplus \mathfrak{g}_{1}}, \psi_{\mathfrak{g}_{3,2}}$ and $\psi_{\mathfrak{g}_{3,3}}$, were already calculated by Novotný and Hrivnák in [18]. They are the following:

$$
\begin{aligned}
& \mathfrak{g}_{1}: \text { Abelian Lie algebra } \\
& \mathfrak{g}_{3,1}:\left[e_{2}, e_{3}\right]=e_{1} \\
& \mathfrak{g}_{2,1} \oplus \mathfrak{g}_{1}:\left[e_{1}, e_{2}\right]=e_{2} \\
& \mathfrak{g}_{3,2}:\left[e_{1}, e_{3}\right]=e_{1},\left[e_{1}, e_{3}\right]=e_{1}+e_{2} \\
& \mathfrak{g}_{3,3}:\left[e_{1}, e_{3}\right]=e_{1},\left[e_{2}, e_{3}\right]=e_{2}
\end{aligned}
$$

\begin{tabular}{|c||c|}
\hline$\alpha$ & $\forall \alpha \in \mathbb{C}$ \\
\hline$\psi_{3 \mathfrak{g}_{1}}(\alpha)$ & 9 \\
\hline
\end{tabular}

\begin{tabular}{|c||c|}
\hline$\alpha$ & $\forall \alpha \in \mathbb{C}$ \\
\hline$\psi_{\mathfrak{g}_{3,1}}(\alpha)$ & 6 \\
\hline
\end{tabular}

\begin{tabular}{|c||c|c|}
\hline$\alpha$ & $\forall \alpha \in \mathbb{C} \backslash\{0\}$ & 0 \\
\hline$\psi_{\mathfrak{g}_{2,1} \oplus \mathfrak{g}_{1}}(\alpha)$ & 4 & 6 \\
\hline
\end{tabular}

\begin{tabular}{|c||c|c|}
\hline$\alpha$ & 1 & $\forall \alpha \in \mathbb{C} \backslash\{1\}$ \\
\hline$\psi_{\mathfrak{g}_{3,2}}(\alpha)$ & 4 & 3 \\
\hline
\end{tabular}

\begin{tabular}{|c||c|c|}
\hline$\alpha$ & 1 & $\forall \alpha \in \mathbb{C} \backslash\{1\}$ \\
\hline$\psi_{\mathfrak{g}_{3,3}}(\alpha)$ & 6 & 3 \\
\hline
\end{tabular}

As $\psi_{\mathfrak{f}_{3}} \leq \psi_{3 \mathfrak{g}_{1}}$ and $\psi_{\mathfrak{f}_{3}}(1)<\psi_{3 \mathfrak{g}_{1}}(1)$, theorem 2.4 assures the existence of a proper contraction from $\mathfrak{f}_{3}$ to $3 \mathfrak{g}_{1}$. Analogously, the same occurs between $\mathfrak{g}_{3,2}$ and $\mathfrak{f}_{3}$ since $\psi_{\mathfrak{g}_{3,2}} \leq \psi_{\mathfrak{f}_{3}}$ and $\psi_{\mathfrak{g}_{3,2}}(1)<\psi_{\mathfrak{f}_{3}}(1)$.

Moreover, note that $\psi_{\mathfrak{f}_{3}}(1)=6$ and $\psi_{\mathfrak{g}_{3,1}}(1)=6$. Therefore, the same theorem assures that there not exists any proper contraction between $\mathfrak{g}_{3,1}$ and $\mathfrak{f}_{3}$. Similarly, the same occurs between $\mathfrak{g}_{3,3}$ and $\mathfrak{f}_{3}$ due to that $\psi_{\mathfrak{f}_{3}}(1)=6$ and $\psi_{\mathfrak{g}_{3,3}}(1)=6$.

Besides, according to theorem 2.2, the algebras $\mathfrak{g}_{3,1}$ and $\mathfrak{f}_{3}$ are isomorphic. This implies that there is not any proper contraction between themselves.

\subsection{Proper contractions between Heisenberg algebras and filiform Lie algebras}

Since that, by definition, there not exists any proper contraction between $\mathfrak{g}_{3,1}$ and $\mathfrak{f}_{3}$, we now ask ourselves if there exists a proper contraction between a Heisenberg algebra and a filiform Lie algebra in the case of dimension five.

To deal with this question, let us consider the Heisenberg algebra of dimension 5, defined by the brackets $\left[e_{1}, e_{3}\right]=e_{5}$ and $\left[e_{2}, e_{4}\right]=e_{5}$.

We want to obtain a basis of $\operatorname{Der}_{(\alpha, 1,1)} \mathbb{H}_{5} \forall \alpha \in \mathbb{C}$. To do this, let us consider $d \in \operatorname{Der}_{(\alpha, 1,1)} \mathbb{H}_{5}$ and the associated matrix with the endomorphism $d$

$$
\left(\begin{array}{llll}
a_{11} & a_{12} & a_{13} & a_{15} \\
a_{21} & a_{22} & a_{23} & a_{25} \\
a_{31} & a_{32} & a_{33} & a_{35} \\
a_{41} & a_{42} & a_{43} & a_{45} \\
a_{51} & a_{52} & a_{53} & a_{55}
\end{array}\right) .
$$


By proceeding in the same way as in the subsection 3.1, we obtain the following conditions for the elements of the matrix

\begin{tabular}{|c|l|}
\hline From pair $\left(e_{i}, e_{j}\right)$ & Conditions \\
\hline \hline$\left(e_{1}, e_{2}\right)$ & $a_{14}=a_{23}$. \\
\hline$\left(e_{1}, e_{3}\right)$ & $\alpha a_{51}=0, \quad \alpha a_{52}=0, \quad \alpha a_{53}=0$, \\
& $\alpha a_{54}=0, \quad \alpha a_{55}=a_{11}+a_{33}$. \\
\hline$\left(e_{1}, e_{4}\right)$ & $a_{12}=-a_{43}$. \\
\hline$\left(e_{1}, e_{5}\right)$ & $a_{53}=0$. \\
\hline$\left(e_{2}, e_{3}\right)$ & $a_{21}=-a_{34}$. \\
\hline$\left(e_{2}, e_{4}\right)$ & $a_{11}+a_{33}=a_{22}+a_{44}$. \\
\hline$\left(e_{2}, e_{5}\right)$ & $a_{54}=0$. \\
\hline$\left(e_{3}, e_{4}\right)$ & $a_{32}=a_{41}$. \\
\hline$\left(e_{3}, e_{5}\right)$ & $a_{51}=0$. \\
\hline$\left(e_{4}, e_{5}\right)$ & $a_{52}=0$. \\
\hline
\end{tabular}

This implies that

$$
\operatorname{dim}\left(\operatorname{Der}_{(\alpha, 1,1)} \mathbb{H}_{5}\right)=15, \forall \alpha \in \mathbb{C}
$$

and thus

\begin{tabular}{|c||c|}
\hline$\alpha$ & $\forall \alpha \in \mathbb{C}$ \\
\hline$\psi_{\mathbb{H}_{5}}(\alpha)$ & 15 \\
\hline
\end{tabular}

So, as $\psi_{\mathbb{H}_{5}}>\psi_{f_{5}}$, Theorem 2.4 proves that there not exists any proper contraction between a Heisenberg algebra and a filiform Lie algebra, both of dimension 5. This result will be commented in the following section.

\section{Conclusions}

In this paper we have studied the proper contractions of filiform Lie algebras of lower dimensions by using a tool previously introduced in 2007 by Hrivnák and Novotný [17]: the invariant functions $\psi$ and $\varphi$, which we have particularized to the case of such algebras. Recall that our motivation for dealing with filiform Lie algebras has been explained in the Introduction of this paper.

Indeed, although by using a different procedure, we have confirmed some results by those authors and have also obtained new results in the case of filiform Lie algebras of dimension 5. In our opinion this is a small improvement since this dimension is not studied in detail in [18] (in fact, the word filiform is not used by the authors in their paper).

Furthermore, in our manuscript we have shown several examples that could be of potential interest for the treatment of other nilpotent Lie algebras different to the filiform or Heisenberg ones. Note that we have also given the procedure and computed the invariant functions for other types of Lie algebras, as the ones built by means of direct sums of other Lie algebras, for instance.

We have also calculated the invariant function $\psi$ for the five dimensional Heisenberg algebra and have proved that there is no proper contraction from a lower dimensional filiform Lie algebra to a Heisenberg algebra. Furthemore, because neither these algebras are isomorphic, then it can not exist a non proper contraction between them. Therefore, we can conclude that for a 5-dimensional 
classical-mechanical model built upon a 5-dimensional filiform Lie algebra can not be obtained as a limit process of a quantum-mechanical model based on a 5-dimensional Heisenberg algebra.

With respect to invariant functions $\psi$ and $\varphi$, we have found that for the $\psi$ invariant function the dimension is always the same independently of the value of $\alpha$, which does not occur in the case of the $\varphi$ invariant function. This feature occurs at least for lower dimensional filiform Lie algebras. We think that it could be interesting to check whether this is a general characteristic irrespective of the dimension since that, at present, we do not know exactly which is the meaning of this fact. One reason could be that both invariant functions are not comparable because they are defined in a totally different way, since the function $\psi$ is defined as the dimension of a derivation whereas $\varphi$ is defined as the dimension of a cocycle, which could involve that their computations were completely different.

And with respect to contractions, we have proved that there is a proper contraction from the filiform Lie algebra $\mathfrak{f}_{3}$ both to the algebra $3 \mathfrak{g}_{1}$ and the algebra $\mathfrak{g}_{3,2}$, whereas it exists no proper contraction either between $\mathfrak{f}_{3}$ and $\mathfrak{g}_{3,1}$, or $\mathfrak{f}_{3}$ and $\mathfrak{g}_{3,3}$.

Remember that, nowadays, the contractions of algebras is a hot topic in Mathematical Physics as we can check in a quick search on the literature. Basically, if two physical theories are related by a limiting process then the associated invariance groups should also be related by some limiting process called contraction. The main example can be found in the Galilean group, which is a contraction of the Poincarè group, that is, classical mechanics is the limiting case of relativistic mechanics as we pointed our in the Introduction of the manuscript. Apart from this example, another physical systems are being explored as we can see in [19] and references therein. In that paper, it is explored the contraction of a $W_{3}$ algebra, which is a nonlinear $W$-algebra, in the context of a Conformal Field Theory. $W$-algebras can be regarded as generalizations of affine Kac-Moody algebras and Virasoro algebra.

Finally, we would like to say that our intention is to deal with the invariant function $\varphi$ for filiform Lie algebras of greater dimensions in future work. Indeed, we are now trying to model a Bose-Hubbard-like model for describing interacting spinless bosons on a lattice by means a filiform Lie algebra. That is a nonlinear problem that if we succeed, it could be mapped to a system with one-body interactions, being therefore linear. Studies of invariance groups in that kind of system and their connection with contractions it is also something worth exploring.

\section{Acknowledgments}

This work has been partially supported by the Spanish Ministerio de Ciencia e Innovación, Grants No FIS 2011-28738-C02-01, FIS2014-53448-C2-1-P, MTM2010-19336 and FEDER, and by Junta de Andalucía, Grants No FQM-160 and FQM-326. Apart from that, the authors want to thank professor J.Hrivnák for their useful comments and suggestions.

\section{References}

[1] L. Boza, E.M. Fedriani, J. Núñez and A.F. Tenorio, A historical review of the classifications of Lie algebras, Revista de la Unión Matemática Argentina 54:2 (2013), 75-99.

[2] D. Burde, Degenerations of nilpotent Lie algebras, J. Lie Theory 9 (1999), 193-202.

[3] D. Burde, Degenerations of 7-dimensional nilpotent, Comm. Algebra 33 (2005), 1259-1277.

[4] H.D. Doebner and O. Melsheimer, On a class of generalized group contractions, Nuovo Cimento A 49:10 (1967), 306-311. 
[5] F.J. Echarte, J. Núñez and F. Ramírez, Description of some families of filiform Lie algebras, Houston Journal of Mathematics 34:1 (2008), 19-32.

[6] A. Fialowski and M. de Montigny, Contractions and deformations of Lie algebras in Physics, American Institute of Physics 38 (2005), 6335-6349.

[7] E. Frenkel and D. Ben-Zvi, Vertex algebras and algebraic curves, Mathematical surveys and monographs 88, Second edition. American Mathematical Society, 2004.

[8] J.E. Humphreys, Introduction to lie algebras and representation theory. Springer-Verlag, New York, 1972.

[9] E. Inönü and E. Wigner, On the contraction of groups and their representations, Proc. Nat. Acad. Sci. U.S.A. 39 (1953), 510-524.

[10] E. Inönü and E. Wigner, On a particular type of convergence to a singular matrix, Proc. Nat. Acad. Sci. U.S.A. 40 (1954), 119-121.

[11] A.A. Izmestév, G.S. Pogosyan, A.N. Sissakian, P. Winternitz, Contractions of Lie algebras and separation of variables, Journal of Physics A: Mathematical and General 29:18 (1996), 5949-5962.

[12] J. Hrivnák, Doctoral Thesis: Invariants of Lie algebras. Czech Technical University in Prague, Faculty of Nuclear Sciences and Physical Engineering, 2007.

[13] J. Hrivnák, P. Novotný, J. Patera, J. Tolar, Graded contractions of the Pauli graded sl(3,C), Linear Algebra Applications 418: 2-3 (2006), 498-550.

[14] M. de Montigny, J. Patera, Discrete and continuous graded contractions of Lie algebras and superalgebras, Journal of Physics A: Mathematical and General 24:3 (1991), 525-547.

[15] M. Nesterenko and R. Popovych, Contractions of low-dimensional Lie algebras Journal of Mathematical Physics 47 (2006). doi: 10.1063/1.2400834

[16] P. Novotný, Graded contractions of representations of Lie algebras, Journal of Physics: Conference Series 343 (2012), conference 1.

[17] P. Novotný and J. Hrivnák, On $(\alpha, \beta, \gamma)$-derivations of Lie algebras and corresponding invariant functions, Journal of Geometry and Physics 58:2 (2008), 208-217

[18] P. Novotný and J. Hrivnák,, Twisted cocycles of Lie algebras and corresponding invariant functions, Linear Algebra and its Applications 430:4 (2009), 1384-1403.

[19] J. Rasmussen and C. Raymond, Galilean contractions of W-algebras, Nuclear Physics B 922 (1917), 435-476.

[20] E.J. Saletan, Contraction of Lie groups, J. Math. Phys. 2 (1961), 1-21.

[21] I.E. Segal, A class of operator algebras which are determined by groups, Duke Math. J. 18 (1951), 221-265.

[22] M. Vergne, Cohomologie des algèbres de Lie nilpotentes, Application à l'étude de la variété des algebres de Lie nilpotentes. Bull. Soc. Math. France 98 (1970), 81-116.

[23] E. Weimar-Woods, Contractions of Lie algebras: Generalized Inönü-Wigner contractions versus graded contractions, Journal of Mathematical Physics 36 (1995), 4519-4548.

[24] E. Weimar-Woods, The general structure of G-graded contractions of Lie algebras, I: The classification, Canadian Journal of Mathematics 58:6 (2006), 1291-1340.

[25] E. Weimar-Woods, The general structure of G-graded contractions of Lie algebras, II: The contracted Lie algebra, Reviews in Mathematical Physics 18:6 (2006), 655-711. 